Pacific Journal of Mathematics

CHARACTERIZATION OF A FUNCTION BY CERTAIN 


\section{CHARACTERIZATION OF A FUNCTION BY CERTAIN INFINITE SERIES IT GENERATES}

\section{Charles K. Chui and Philip W. Smith}

Let $A$ be a set of real numbers and $F$ be a class of complex-valued functions defined on the real line such that for each $f \in F$ the infinite series $S(x, f)=\sum_{k=1}^{\infty} f(k x)$ converges for every nonzero $x$ in $A$. If $0 \in A$, we set $S(0, f)=f(0)$. It seems to be an interesting problem to study the different sets $A$ and function classes $F$ such that each $f \in F$ is uniquely determined by the sums $S(x, f)$ where $x \in A$. Clearly, the larger the class $F$ is studied, the larger set $A$ is needed to guarantee uniqueness. We have positive results for a class of entire functions of exponential type and for fairly large classes of continuous functions. Some examples are also given to show that in general $A$ cannot be too small.

1. Introduction. For a function $f$ holomorphic in the open unit disc $U$ of the complex plane and continuous on the closure of $U$, let

$$
s_{n}(f, \delta)=\frac{1}{n} \sum_{k=1}^{n} f\left(e^{i 2 \pi \delta k / n}\right), \quad n=1,2, \cdots, 0<\delta \leqq 1 .
$$

Sufficient conditions on the function $f$ were given in $[2,5]$ to guarantee that $f$ is uniquely determined by the means $s_{n}(f, 1)$, and in [3] both positive and negative results were given for the case $0<\delta<1$. The annulus case was also studied in [4]. In this paper we study a related problem for an unbounded interval.

Let $A$ be a set of real numbers and $F$ be a class of complexvalued functions defined on the real line $R$ such that for each $f \in F$, the infinite series $S(x, f)=\sum_{k=1}^{\infty} f(k x)$ converges for every nonzero $x \in A$. If $0 \in A$, we denote $S(0, f)=f(0)$. We study various sets $A$ and various function classes $F$ such that each $f \in F$ is uniquely determined by the sums $S(x, f)$ where $x \in A$. Some examples will be given to show that in general $A$ cannot be too small.

2. Results for entire functions. We start with a fairly small function class. For $r>0$, let $P W(r \pi, 1)$ be the class of all entire functions $f(z)$ of exponential type at most $r \pi$ such that $f(x) \in L^{2}(R)$ and that for some $p>1, f(n / r)=0\left(|n|^{-p}\right)$ for $n= \pm 1, \pm 2, \cdots$. Let $Z$ be the set of all integers. We have the following theorem of the Carlson type.

THEOREM 1. Every function $f$ in $P W(\pi, 1)$ is uniquely determined by the sequence $S(n, f)$ where $n \in Z$. 
We remark that the above result is in a sense sharp in that every $S(n, f), n \in Z$, is needed to determine $f$. That is, we have the following

Lemma 1. For each $n \in Z$, there is a unique function $f_{n} \in P W(\pi, 1)$ such that $S\left(m, f_{n}\right)=\delta_{m, n}$ for all $m \in Z$, where $\delta_{m, n}$ is the Kronecker delta.

Let $\mu(n)$ denote the Möbius function (cf. [8]), that is,

$$
\mu(n)= \begin{cases}1 & \text { if } n=1 \\ (-1)^{k} & \text { if } n=p_{1} \cdots p_{k} \text { with distinct primes } p_{j} \\ 0 & \text { if } p^{2} \mid n \text { for some } p>1 .\end{cases}
$$

We can determine the functions $f_{n}$ in the above lemma explicitly as in the following

LEMma 2.

$$
\begin{array}{ll}
f_{0}(z)=\frac{\sin \pi z}{\pi z}, & \text { and for } n=1,2, \cdots, \\
f_{n}(z)=\sum_{k \mid n} \mu\left(\frac{n}{k}\right) \frac{\sin \pi(z-k)}{\pi(z-k)}, & \text { and for } n=-1,-2, \cdots, \\
f_{n}(z)=f_{-n}(-z) . &
\end{array}
$$

Of course, at the removable singular points these functions are defined so that they are entire. It is then clear that they are in the class $P W(\pi, 1)$. By using the equations

$$
\sum_{k \in n} \mu\left(\frac{n}{k}\right)=\delta_{n, 1},
$$

it is not difficult to show that they satisfy the relations $S\left(m, f_{n}\right)=$ $\hat{o}_{m, n}$ for all $m, n \in Z$. Finally, the uniqueness result in Theorem 1 guarantees that they must be the functions in Lemma 1. By using this sequence of functions $f_{n}$, we can actually construct each function $f \in P W(\pi, 1)$ from the corresponding sequence $S(n, f), n \in Z$, as in the following

Theorem 2. Let $f \in P W(\pi, 1)$. Then

$$
f(z)=\sum_{n=-\infty}^{\infty} S(n, f) f_{n}(z)
$$

where the infinite series converges uniformly on each horizontal strip $|\operatorname{Im} z| \leqq K<\infty$ of the complex plane.

To prove Theorem 1, we let $f \in P W(\pi, 1)$ such that $S(m, f)=0$ 
for all $m \in Z$. That is $f(0)=0$,

$$
\sum_{k=1}^{\infty} f(k m)=0 \text { and } \sum_{k=1}^{\infty} f(-k m)=0
$$

for all $m=1,2, \cdots$. Since $f(n)=0\left(1 /|n|^{p}\right)$ for some $p>1$ and $n=$ $\pm 1, \pm 2, \cdots$, these infinite series converge absolutely. As in [2] we multiply $\mu(m)$ to the first series in (2) and sum it from 1 to $N$ to obtain

$$
f(1)=-\sum_{j=N+1}^{\infty}\left(\sum_{n \mid j}^{1 \leqq n \leqq N} \mu(n)\right) f(j) \text {. }
$$

Let $d(m)$ be the number of divisors of $m$ and it is known (cf. [8]) that $d(m)=O\left(m^{i}\right)$ for all positive $\delta$. Pick $\delta=(p-1) / 2$. Then from (3), we get

$$
\begin{aligned}
|f(1)| & \leqq \sum_{j=N+1}^{\infty} \sum_{n \mid j}|\mu(n)||f(j)| \\
& \leqq \sum_{j=N+1}^{\infty} d(j)|f(j)|=O\left(N^{-(p-1) / 2}\right) .
\end{aligned}
$$

Hence, $f(1)=0$. For each fixed $k \geqq 1$, we let $a_{j}=f(j k)$ and note that $\sum_{v=1}^{\infty} a_{j v}=0$. This gives $f(k)=a_{1}=0$. Hence, $f(k)=0$ for $k=1,2, \cdots$ Applying the same proof to the second series in (2), we have $f(k)=0$ for $k=-1,-2, \cdots$. Next, by the Paley-Wiener theorem (cf. [1]) we can write

$$
f(z)=\int_{-\pi}^{\pi} e^{i z t} g(t) d t
$$

where $g \in L^{2}(-\pi, \pi)$. Since $f(k)=0$ for all $k \in Z$, all the Fourier coefficients of $g$ vanish, so that $g=0$ a.e., or $f$ is the zero function. This completes the proof of Theorem 1.

To prove Theorem 2, we let $K$ be any positive number, and by a Phragmen-Lindelof theorem, we note that $|\sin \pi z / \pi z| \leqq e^{\pi K} / \pi K=C_{K}$ for all $z$ in the strip $|\operatorname{Im} z| \leqq K$. Hence, for $|\operatorname{Im} z| \leqq K$,

$$
\left|f_{n}(z)\right| \leqq C_{k} \sum_{k \mid n}\left|\mu\left(\frac{n}{k}\right)\right| \leqq C_{K} d(n) \text {. }
$$

Let $f \in P W(\pi, 1)$. Then $f(n)=O\left(|n|^{-p}\right)$ for some $p>1$ as $|n| \rightarrow \infty$. Hence, for $n= \pm 1, \pm 2, \cdots$

$$
|S(n, f)| \leqq \sum_{k=1}^{\infty}|f(k n)| \leqq c_{1} \sum_{k=1}^{\infty} k^{-p}|n|^{-p}=c_{2}|n|^{-p} .
$$

Pick $\delta=(p-1) / 2$. Then for all $z$ in the strip $|\operatorname{Im} z| \leqq K$, we have $\left|S(n, f) f_{n}(z)\right| \leqq c_{3}|n|^{-p} d(|n|) \leqq C_{K, \dot{o}}|n|^{-(p+1) / 2}$, so that the series 


$$
\sum_{n=-\infty}^{\infty} S(n, f) f_{n}(z)
$$

converges uniformly on every horizontal strip $|\operatorname{Im} z| \leqq K<\infty$ to an entire function $f^{*}(z)$. Next, we consider the sequence

$$
g_{n}(t)=\frac{1}{2 \pi} S(n, f) \sum_{k i n} \mu\left(\frac{n}{k}\right) e^{-i k t} .
$$

By the same estimate as above and by the Weierstrass $M$-test, the series $\sum_{n=1}^{\infty} g_{n}(t)$ converges uniformly to a continuous function $g(t)$ on $[-\pi, \pi]$. Hence,

$$
\begin{aligned}
\int_{-\pi}^{\pi} e^{i t z} g(t) d t & =\sum_{n=1}^{\infty} \int_{-\pi}^{\pi} e^{i t z} g_{n}(t) d t \\
& =\sum_{n=1}^{\infty} S(n, f) \sum_{k \mid n} \mu\left(\frac{n}{k}\right) \frac{1}{2 \pi} \int_{-\pi}^{\pi} e^{i(z-k) t} d t \\
& =\sum_{n=1}^{\infty} S(n, f) \sum_{k \mid n} \mu\left(\frac{n}{k}\right) \frac{\sin \pi(z-k)}{\pi(z-k)} \\
& =\sum_{n=1}^{\infty} S(n, f) f_{n}(z) .
\end{aligned}
$$

Therefore, we have

$$
f^{*}(z)=\int_{-\pi}^{\pi} e^{i t z}(g(t)+g(-t)) d t+f(0) .
$$

By the Paley-Wiener theorem (cf. [1], p. 103) we can conclude that $f^{*}$ is an entire function of exponential type at most $\pi$ and belongs to $L^{2}(R)$ on the real axis. For positive integers $n$, it is not difficult to show that

$$
\begin{aligned}
\left|f^{*}(n)\right| & =\left|\sum_{k=1, n \mid k}^{\infty} \mu\left(\frac{k}{n}\right) S(k, f)\right| \\
& =\left|\sum_{k=1}^{\infty} \mu(k) S(k n, f)\right| \leqq \sum_{k=1}^{\infty}|S(k n, f)| \\
& \leqq C_{1} \sum_{k=1}^{\infty} k^{-p} n^{-p}=O\left(n^{-p}\right) .
\end{aligned}
$$

This also holds for negative integers $n$. That is, we have proved that $f^{*} \in P W(\pi, 1)$. Now, since $S\left(n, f^{*}\right)=\sum_{k=-\infty}^{\infty} S(k, f) S\left(n, f_{k}\right)=$ $S(n, f)$ for all $n \in Z$, we have $f=f^{*}$ by Theorem 1 , and thus, the proof of Theorem 2 is completed.

3. Examples and counterexamples. In this section we will present some examples which will be used to indicate the sharpness of results to follow. The first proposition deals with entire functions of exponential type. 
Proposition 1. Every function $f \in P W(r \pi, 1), r>0$, is uniquely determined by the sequence $S(x, f)$ where $x \in r^{-1} Z$; furthermore $r^{-1} Z$ cannot be reduced.

The next three propositions deal with more general sets $A$ and functions defined on the interval $[0, \infty)=R^{+}$. If $A$ is bounded below, we have

Proposition 2. If $0<\delta=\inf \{x: x \in A\}$ then every $f$ with

$$
(\operatorname{supp} f) \subset[0, \delta)
$$

satisfies $S(x, f)=0$ for all $x \in A$.

If $A$ is bounded above we obtain

Proposition 3. If $\Delta=\sup \{x: x \in A\}<\infty$ then there is a nontrivial function $f \in P W(2 \pi / \Delta, 1)$ satisfying $S(x, f)=0$ for all $x \in A$.

Finally we show that $A$ may contain an interval about the origin and also be unbounded or $A$ may be a neighborhood of infinity and contain a sequence converging to zero. Let $S$ be the space of complexvalued functions $f$ which are in $C^{\infty}$ and for any $n>0,|f(x)||x|^{n}=$ $O(1)$ as $|x| \rightarrow \infty$, and let $Z^{+}$be the set of positive integers. We have

Proposition 4. There is a nontrivial function $f \in S$ whose cosine transform $f_{c}$ is also in $S$ satisfying $S(x, f)=0$ for all $x \in A=$ $[0,1 / 2] \cup Z^{+}$and $S\left(x, f_{c}\right)=0$ for all $2 \pi / x \in A$.

We turn now to the proof of Proposition 1. Actually Proposition 1 requires little work since it can be seen that this is just a restatement of Theorem 1 and Lemma 1 with a change of variable. The important point in Proposition 1 is that as $A=r^{-1} Z$ becomes larger, i.e., $r$ gets larger, the sums characterize a larger function class.

Proposition 2 is clear since all the sums vanish identically. We will soon show that this proposition is in a sense dual to Proposition 3. The duality is due to the Poisson summation formula. Let $f \in$ $L^{1}\left(R^{+}\right)$and following [10] define $f_{\mathrm{c}}$ the cosine transform of $f$ to be

$$
f_{c}(t)=\sqrt{\frac{2}{\pi}} \int_{R^{+}} f(x) \cos x t d x .
$$

If $f$, in addition, is continuous and $\{f(n \alpha)\}_{n=0}^{\infty} \in l^{1}$ for $\alpha>0$ then

$$
\beta^{1 / 2}\left[\frac{1}{2} f_{c}(0)+\sum_{n=1}^{\infty} f_{c}(n \beta)\right]=\alpha^{1 / 2}\left[\frac{1}{2} f(0)+\sum_{n=1}^{\infty} f(n \alpha)\right]
$$

where $\alpha \beta=2 \pi$. This formula is known as the Poisson summation 
formula.

If $f$ has compact support in

$$
(0,2 \pi / \Delta), \int_{R^{+}} f=0 \text {, and } A \cap[0,2 \pi / \Delta)=\varnothing,
$$

then Proposition 1 tells us that $S(x, f)=0$. Suppose $f$ is smooth enough (say $C^{2}$ ) so that $f_{c} \in P W(2 \pi / \Delta, 1)$. Then, by the Poisson summation formula we have $S\left(2 \pi / x, f_{c}\right)=0$ for all $x \in A$. Note that $\sup \{2 \pi / x: x \in A\} \leqq \Delta$, and this proves Proposition 3 .

Thus, if we wish to recapture a large class of functions from their sums we must have a set $A$ which is unbounded and contains a sequence converging to 0 . Proposition 4 shows that we even need much more than that. Let $f$ have a cosine transform $f_{c}$ which is in $C^{\infty}$ and has compact support in $(0,4 \pi)$ (hence $f_{c} \in S$ ). Suppose $\int_{R^{+}} f_{c}=0$ and $f_{c}$ is odd about $2 \pi$, then $S\left(x, f_{c}\right)=0$ for all $2 \pi / x \in A$ and then by the Poisson summation formula $S(x, f)=0$ for all $x \in A$. Furthermore, $f \in S$ since the Fourier transform is an $L^{2}(R)$ isomorphism of $S$ onto $S$.

4. Results for larger classes. Let $W^{1,1}\left(R^{+}\right)$be the Sobolev space of functions in $L^{1}\left(R^{+}\right)$which are absolutely continuous and whose derivatives are in $L^{1}\left(R^{+}\right)$. In [9] it was shown that for any $f \in W^{1,1}(R)$, $S(x, f)$ is absolutely convergent for any $x \neq 0$. Of course, a similar statement is true for $f \in W^{1,1}\left(R^{+}\right)$and $x>0$. We have the following result.

Theorem 3. Let $A=\left\{L_{j} / n: n=1,2, \cdots, 0=L_{1}<L_{2}<\cdots\right\}$ then the set of functionals $\{S(x, \cdot): x \in A\}$ is total over $W^{1,1}\left(R^{+}\right)$if and only if the sequence $\left\{L_{j}\right\}$ is unbounded.

If the $L_{i}$ are bounded above, then Proposition 3 tells us that $\{S(x, \cdot): x \in A\}$ is not total. The converse is a bit more difficult. Suppose $\left\{L_{i}\right\}_{i=1}^{\infty}$ is unbounded, and suppose $f$ is in $W^{1,1}\left(R^{+}\right)$and satisfies $S(x, f)=0$ for all $x \in A$. We must show that $f \equiv 0$. We first observe that $\int_{R^{+}} f=0$. Indeed, for any $h>0$ we have

$$
\begin{aligned}
\left|\int_{0}^{\infty} f(t) d t-h S(h, f)\right| & =\left|\int_{0}^{\infty}\left(f(t)-\sum_{k=1}^{\infty} \chi_{[(k-1) h, k h]}(t) f(k h)\right) d t\right| \\
& \leqq \sum_{k=1}^{\infty} \int_{(k-1) h}^{k h}|f(t)-f(k h)| d t \\
& =\sum_{k=1}^{\infty} \int_{(k-1) h}^{k h}\left|\int_{k h}^{t} f^{\prime}(\tau) d \tau\right| d t \\
& \leqq\left|h \int_{0}^{\infty}\right| f^{\prime} \mid .
\end{aligned}
$$


Let $\left\{h_{j}\right\}$ be a sequence in $A$ with $h_{j} \rightarrow 0$. Setting $h=h_{j}$ in (4) yields the result.

Let $\nu(t)$ be the "saw-toothed" function defined by

$$
\nu(t)=\left\{\begin{array}{cc}
{[t]-t+\frac{1}{2}} & t \notin Z \\
0 & t \in Z .
\end{array}\right.
$$

For any $x \in A, x \neq 0$, and $f$ as above, we have as in [5]

$$
\begin{aligned}
0 & =S(x, f)=\sum_{k=1}^{\infty} f(k x) \\
& =-\int_{0}^{\infty} \nu(t / x) f^{\prime}(t) d t+\frac{1}{x} \int_{0}^{\infty} f(t) d t-\frac{1}{2} f(0) \\
& =-\int_{0}^{\infty} \nu(t / x) f^{\prime}(t) d t .
\end{aligned}
$$

In $[6,7]$ Davenport established

$$
\sin 2 \pi n t=\lim _{N \rightarrow \infty} \sum_{k=1}^{N} \frac{\mu(k)}{k} \pi \nu(n k t)
$$

where the convergence is uniform. Now for any $x \in A, x / k \in A$. Hence, if $x \neq 0, x \in A$, we have by (5)

$$
\begin{aligned}
0 & =\lim _{N \rightarrow \infty} \sum_{k=1}^{N} \frac{\mu(k)}{k} \pi \int_{0}^{\infty} \nu(k t / x) f^{\prime}(t) d t \\
& =\int_{0}^{\infty}[\sin (2 \pi t / x)] f^{\prime}(t) d t .
\end{aligned}
$$

But this means that the Fourier sine transform of $f^{\prime}$ vanishes at all the points $2 \pi / x, x \in A$. Since this set is dense on $R^{+}$and $f^{\prime} \in L^{1}\left(R^{+}\right)$ we conclude that $f^{\prime} \equiv 0$. Hence, $f \equiv 0$ which was to be shown.

It is interesting to note the algebraic nature of the set $A$ in Theorem 3. In order to use Davenport's result, we needed to have $k A^{-1} \subset A^{-1}$ for every positive integer $k$. Along these same lines we have

LEMMA 3. Let $f$ be a bounded function from $R^{+}$into $R$ satisfying $f(x)=O\left(x^{-p}\right)$ for some $p>1$, as $x \rightarrow \infty$. Let $A=(\delta, \infty), \delta \geqq 0$, then the values $S(x, f), x \in A$, uniquely determine $f$ on $(\delta, \infty)$.

For suppose that $S(x, f)=0, x \in A$, then we obtain the infinite homogeneous linear system

$$
0=S(n x, f)
$$$$
n=1,2, \cdots
$$ 
As seen in the proof of Theorem 1, this system has only the trivial solution, hence $f(x)=0$ for all $x \in A=(\delta, \infty)$. This lemma is remarkable in that very few restrictions were put on the functions $f$. In fact, $f$ may be nonmeasurable. Of course, as a corollary we obtain

CoRollary 1. If $f$ is as in Lemma 3 and $f$ is continuous, then $S(x, f), x \in A$, uniquely determines $f$ on $(\delta, \infty)$ where $A$ need only be a dense subset of $(\delta, \infty)$ satisfying $k A \subset A$ for all $k \in Z^{+}$.

In all the above results of this section we have needed the density of the determining set $A^{-1}$ in $R^{+}$(as in Theorem 3) or the whole interval $(\delta, \infty)$ when we determine the functions on $(\delta, \infty)$. Furthermore, Propositions 2, 3, and 4 seem to indicate that $A$ must be large in order to characterize large function classes. However, we have the following

LEMMA 4. If $f \in C^{2}\left(R^{+}\right), f(x)=O\left(x^{-p}\right)$ for some $p>1$, as $x \rightarrow \infty$, and $f^{\prime}(0)=0$, then $f$ is uniquely determined by the sums $S(x, f)$ for all $x$ in

$$
A=\left[0, \delta_{1}\right) \cup\left(\delta_{2}, \infty\right)
$$

where $0<\delta_{1}<\delta_{2}$.

Suppose $S(x, f)=0, x \in A$. Then by Lemma 3 we know that $(\operatorname{supp} f) \subset\left[0, \delta_{2}\right]$. Let, as before, $f_{c}$ be the cosine transform of $f$. Due to the regularity of $f$ we see that $f_{c}$ also satisfies the hypotheses of Lemma 3. Furthermore, the Poisson summation formula yields

$$
0=S(x, f)=S\left(\frac{2 \pi}{x}, f_{c}\right)
$$

for all $x \in\left(0, \delta_{1}\right)$. Lemma 3 then implies that $f_{c}$ vanishes identically in $\left(2 \pi / \delta_{1}, \infty\right)$. But $f_{c}$ is entire so $f_{c} \equiv 0$. Thus $f \equiv 0$ which was to be proved.

Comparing Lemma 4 to Proposition 4 it is clear that the hypotheses can be relaxed only slightly since the functions in Proposition 4 can be chosen to satisfy the hypotheses of Theorem 4 . We do have the following

TheOREm 4. Let $f$ be as in Lemma 4, and for $\delta>0$ let

$$
A=(\delta, \infty) \cup\left\{2 \delta / k: k \in Z^{+}\right\} \text {. }
$$

Then $f$ is uniquely determined by the sums $S(x, f), x \in A$. 
As usual we assume that $S(x, f)=0, x \in A$, and conclude by Lemma 3 that $(\operatorname{supp} f) \subset[0, \delta]$. It follows that $f_{c}$ is entire of exponential type at most $\delta$. By the Poisson summation formula

$$
S\left(x, f_{c}\right)=0
$$

for $x \in\{k \pi / \delta\}, k=1,2, \cdots$. Integration by parts shows that $f_{c}(x)=$ $O\left(x^{-2}\right)$ as $x \rightarrow \infty$, hence $f_{c} \in P W(\delta, 1)$. Since $f_{c}$ is even we have by (7) $S\left(k \pi / \delta, f_{c}\right)=0$ for $k= \pm 1, \pm 2, \cdots$. By a change of variable as in Proposition 1 we conclude by Theorem 2 that $f_{c}(z)=a \sin \delta z / \delta z$. The constant $a$ must be zero since $f_{c}(x)=O\left(x^{-2}\right)$ as $x \rightarrow \infty$. It follows that $f \equiv 0$.

We finally remark that by Proposition 4 the set $A$ in (6) cannot be replaced by

$$
(\delta, \infty) \cup\left\{2 \delta / k: k \in 4 Z^{+}\right\}
$$

However, if we assume in addition $f \in C^{\infty}$ we can replace $A$ in (6) by the set

$$
(\delta, \infty) \cup\{2 \delta / k: k=N, N+1, \cdots\}
$$

for any positive integer $N$.

\section{REFERENCES}

1. R. P. Boas, Jr., Entire Functions, Academic Press, New York, 1954.

2. C. H. Ching and C. K. Chui, Uniqueness theorems determined by function values at the roots of unity, J. Approximation Theory, 9 (1973), 267-271.

3. — Analytic functions characterized by their means on an arc, Trans. Amer. Math. Soc., 186 (1973), 175-183.

4. - Recapturing a holomorphic function on an annulus, Proc. Amer. Math. Soc., 41 (1973), 120-126.

5. — Approximation of functions from their means, Proc. Symposium on Approximation Theory, Austin, Jan., (1973), Ed. G. G. Lorentz, Academic Press, 1973, 307-313.

6. H. Davenport, On some infinite series involving arithmetical functions, Quarterly J. of Math., 8 (1937), 8-13.

7. - On some infinite series involving arithmetical functions, Quarterly J. of Math., 8 (1937), 313-320.

8. G. H. Hardy and E. M. Wright, An Introduction to the Theory of Numbers, Oxford University Press, Oxford, 1954.

9. P. W. Smith, Characterization of the function class $W^{m, p}$, Proc. Symposium on Approximation Theory, Austin Jan., (1973), Ed. G. G. Lorentz, Academic Press, 1973, 485-490.

10. E. C. Titchmarsh, Introduction to the Theory of Fourier Integrals, 2nd Ed. Oxford University Press, Oxford, 1948.

Received March 25, 1973.

TeXAS A \& M UNIVERSITY 



\section{PACIFIC JOURNAL OF MATHEMATICS}

\section{EDITORS}

RICHARD ARENS (Managing Editor)

University of California

Los Angeles, California 90024

\section{J. DUGUNDJI}

Department of Mathematics University of Southern California Los Angeles, California 90007

D. Gilbarg and J. Milgram

Stanford University

Stanford, California 94305

University of Washington
Seattle, Washington 98105

ASSOCIATE EDITORS
E. F, BECKENBACH
B. H. NEUMANN
F. WOLF
K. Yoshida

\section{SUPPORTING INSTITUTIONS}

\author{
UNIVERSITY OF BRITISH COLUMBIA \\ CALIFORNIA INSTITUTE OF TECHNOLOGY \\ UNIVERSITY OF CALIFORNIA \\ MONTANA STATE UNIVERSITY \\ UNIVERSITY OF NEVADA \\ NEW MEXICO STATE UNIVERSITY \\ OREGON STATE UNIVERSITY \\ UNIVERSITY OF OREGON \\ OSAKA UNIVERSITY
}

\author{
UNIVERSITY OF SOUTHERN CALIFORNIA \\ STANFORD UNIVERSITY \\ UNIVERSITY OF TOKYO \\ UNIVERSITY OF UTAH \\ WASHINGTON STATE UNIVERSITY \\ UNIVERSITY OF WASHINGTON \\ * * * * \\ AMERICAN MATHEMATICAL SOCIETY \\ NAVAL WEAPONS CENTER
}

The Supporting Institutions listed above contribute to the cost of publication of this Journal, but they are not owners or publishers and have no responsibility for its content or policies.

Mathematical papers intended for publication in the Pacific Journal of Mathematics should be in typed form or offset-reproduced, (not dittoed), double spaced with large margins. Underline Greek letters in red, German in green, and script in blue. The first paragraph or two must be capable of being used separately as a synopsis of the entire paper. Items of the bibliography should not be cited there unless absolutely necessary, in which case they must be identified by author and Journal, rather than by item number. Manuscripts, in duplicate if possible, may be sent to any one of the four editors. Please classify according to the scheme of Math. Rev. Index to Vol. 39. All other communications to the editors should be addressed to the managing editor, or Elaine Barth, University of California, Los Angeles, California, 90024.

100 reprints are provided free for each article, only if page charges have been substantially paid. Additional copies may be obtained at cost in multiples of 50 .

The Pacific of Journal Mathematics is issued monthly as of January 1966. Regular subscription rate: $\$ 72.00$ a year (6 Vols., 12 issues). Special rate: $\$ 36.00$ a year to individual members of supporting institutions.

Subscriptions, orders for back numbers, and changes of address should be sent to Pacific Journal of Mathematics, 103 Highland Boulevard, Berkeley, California, 94708.

PUBLISHED BY PACIFIC JOURNAL OF MATHEMATICS, A NON-PROFIT CORPORATION

Printed at Kokusai Bunken Insatsusha (International Academic Printing Co., Ltd.), 270, 3-chome Totsuka-cho, Shinjuku-ku, Tokyo 160, Japan.

Copyright (C) 1973 by Pacific Journal of Mathematics Manufactured and first issued in Japan 


\section{Pacific Journal of Mathematics}

\section{Vol. 53, No. $2 \quad$ April, 1974}

Kenneth Abernethy, On characterizing certain classses of first countable spaces by

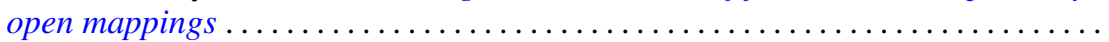

Ross A. Beaumont and Donald Lawver, Strongly semisimple abelian groups .......

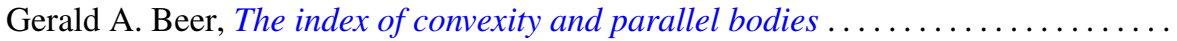

Victor P. Camillo and Kent Ralph Fuller, On Loewy length of rings ..............

Stephen LaVern Campbell, Linear operators for which $T^{*} T$ and $T T^{*}$ commute.

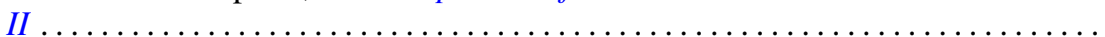

Charles Kam-Tai Chui and Philip Wesley Smith, Characterization of a function by

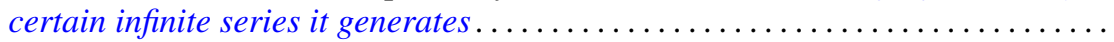

Allan L. Edelson, Conjugations on stably almost complex manifolds . ...........

Patrick John Fleury, Hollow modules and local endomorphism rings . . ..........

Jack Tilden Goodykoontz, Jr., Connectedness im kleinen and local connectedness in

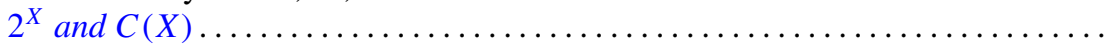

Robert Edward Jamison, II, Functional representation of algebraic intervals .......

Athanassios G. Kartsatos, Nonzero solutions to boundary value problems for

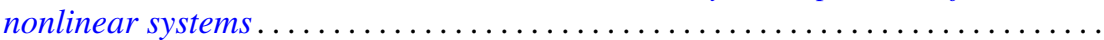

Soon-Kyu Kim, Dennis McGavran and Jingyal Pak, Torus group actions on simply

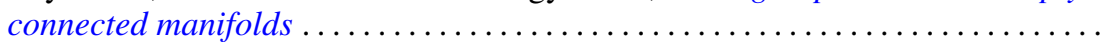

David Anthony Klarner and R. Rado, Arithmetic properties of certain recursively

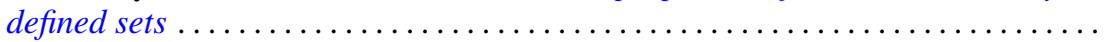

Ray Alden Kunze, On the Frobenius reciprocity theorem for square-integrable

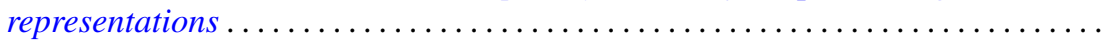

John Lagnese, Existence, uniqueness and limiting behavior of solutions of a class of differential equations in Banach space...

Teck Cheong Lim, A fixed point theorem for families on nonexpansive mappings Lewis Lum, A quasi order characterization of smooth continua

Andy R. Magid, Principal homogeneous spaces and Galois extensions . .

Charles Alan McCarthy, The norm of a certain derivation ..... . .

Louise Elizabeth Moser, On the impossibility of obtaining $S^{2} \times S^{1}$ by elementary surgery along a knot. .

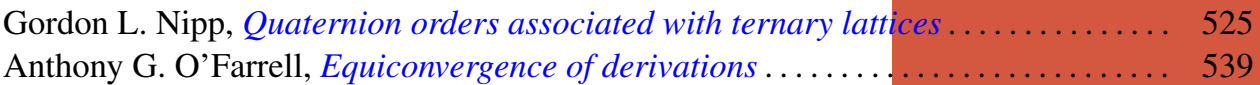

Dorte Olesen, Derivations of $A W^{*}$-algebras are inner . . . . . . . . . . . . . . . 555

Dorte Olesen and Gert Kjærgaard Pedersen, Derivations of $C^{*}$-algebras have

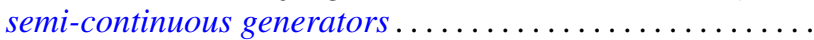

Duane O’Neill, On conjugation cobordism.

Chull Park and S. R. Paranjape, Probabilities of Wiener paths crossing differentiable

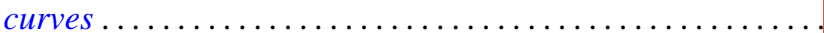

Edward Ralph Rozema, Almost Chebyshev subspaces of $L^{1}(\mu$;

Lesley Millman Sibner and Robert Jules Sibner, A note on the Atiyah-Bott fixed

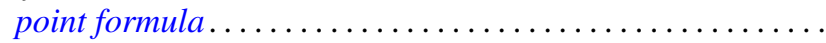

Betty Salzberg Stark, Irreducible subgroups of orthogonal groups generated by

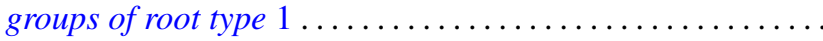

N. Stavrakas, A note on starshaped sets, $(k)$-extreme points and the half ray

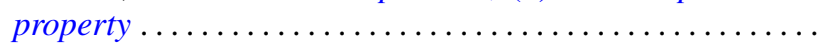

Carl E. Swenson, Direct sum subset decompositions of $Z \ldots \ldots$ 